\title{
20 Jahre vertane deutsche Friedenspolitik - Wir wären gern entschuldigt!
}

\author{
Klaus Schlichtmann*
}

\begin{abstract}
„Things cannot be forced from the top [...]. The international relinquishing of sovereignty would have to spring from the people - it would have to be so strong that the elected delegates would be turned out of office if they failed to do it." (John F. Kennedy) ${ }^{1}$
\end{abstract}

$\mathrm{I}$ m Zwei-plus-Vier-Vertrag, der am 12. September 1990 in Moskau unterzeichnet wurde, hat Deutschland sich dazu verpflichtet, „dem Frieden der Welt zu dienen,“ indem es zusammen mit den anderen Staaten "geeignete Maßnahmen zur Festigung des Weltfriedens" trifft, u.a. um die internationale „Sicherheit zu stärken, insbesondere durch wirksame Maßnahmen zur Rüstungskontrolle, Abrüstung und Vertrauensbildung.“ Von deutschem Boden sollte „Frieden ausgehen“; Waffen sollten nur „in Übereinstimmung mit [dem Grundgesetz] und der Charta der Vereinten Nationen“ eingesetzt werden. ${ }^{2}$ Wie sieht die Bilanz heute aus?

Mit der Unterzeichnung des Vertrags erlangte Deutschland seine volle Souveränität und Einheit. Für viele Beobachter war es damals jedoch keineswegs klar, ob anstelle der Wiedervereinigung nicht eine andere Lösung vorzuziehen gewesen wäre, nämlich eine Art globales Commonwealth. Eine solche Alternative hätte nicht die Nation, sondern eine föderative Ordnung in den Mittelpunkt gerückt, die nicht nur die Länder Europas zusammengefasst hätte, sondern, wie auch in der Charta der Vereinten Nationen (VN) angelegt, über Europa hinausgegangen wäre. ${ }^{3}$ Als Beobachter von damals war für mich der „latente Krieg“4 zu Ende. Nun musste ein globaler Frieden mit einer wesentlich effizienteren UNO und einem echten System kollektiver Sicherheit, gegründet auf internationalem Recht, das Ziel sein.

Stattdessen kam es erstmals seit Ende des Zweiten Weltkriegs in Europa, genauer: in Jugoslawien, wieder zu Krieg und zum ersten Mal in der Geschichte der deutschen Bundeswehr zu einem militärischen Auslandseinsatz. Persönlich hatte ich keine Zweifel, dass die Schaffung einer globalen Friedens- und Rechtsordnung, wie sie von vielen Politikern zum damaligen

* Dr. Klaus Schlichtmann ist Lektor an der Nihon-Universität und Japan Women's University.

1 John F. Kennedy hatte im Juni 1945 als junger Reporter an der Konferenz der Vereinten Nationen in San Francisco teilgenommen und schrieb unter dem Eindruck der Ereignisse dies einem Freund. Arthur M. Schlesinger's A Thousand Days. John F. Kennedy in the White House, New York 2002, S. 88.

2 Vertrag über die abschließende Regelung in bezug auf Deutschland, Bulletin des Presse- und Informationsamtes der Bundesregierung vom 14. September 1990 Nr. 109, S. 1153-1156

3 Eine solche reale Utopie entwirft etwa Hanna Newcombe Design for a better World, Lanham, Maryland 1983.

4 Der Pazifist und Friedensnobelpreisträger Alfred Hermann Fried bezeichnet den Zustand des bewaffneten Friedens als „latenten Krieg. "Fried wendet sich eindeutig gegen die herrschende Auffassung, ,dass der Friede durch Rüstungen geschützt werden könne [...]. Man glaubt, dass die Nichtanwendung der Gewaltmittel bereits den Zustand des Friedens bezeichnet, während es in Wirklichkeit nur der Zustand des Nicht-Krieges oder des latenten Krieges ist." Alfred H. Fried, Das alte und das neue System der Friedenssicherung, in: Vom Weltkrieg zum Weltfrieden. Zwanzig Kriegsaufsätze, Zürich 1916, S. 54.
Zeitpunkt gefordert worden war, die Gewalt und Auseinandersetzungen in Jugoslawien und andernorts hätte verhindern können. Ich möchte behaupten, dass es gar nicht erst zu den Kriegen auf dem Balkan gekommen wäre, wenn der Gesetzgeber in Deutschland rechtzeitig Schritte unternommen hätte, die VN mit den in der Charta vorgesehenen Vollmachten auszurüsten.

Denn die Wiedervereinigung verlangte von der Bundesrepublik die rasche Umsetzung einer neuen Friedens- und Sicherheitspolitik. Das Gebot der Stunde und der „Test für [Deutschlands] Bereitschaft, die in der Charta niedergelegten Rechte und Pflichten voll wahrzunehmen ", 5 war die Umsetzung des Friedensgebots. Entgegen den Vorgaben im Grundgesetz und den Bestimmungen in der VN-Charta beschritt die Bundesrepublik jedoch einen anderen Weg: Anstatt der UNO nationale Hoheitsrechte abzutreten und einen Weltabrüstungsvertrag einzufordern, wie u.a. die Artikel 24 und 26 (VN-Charta und GG) nahelegen, entschied man sich für militärische Auslandseinsätze - trotz der erwiesenen Nachteile und Gefahren militärischer Friedenssicherung. Die damit verbundene Weichenstellung ${ }^{6}$ hatte weitreichende Folgen. Die Verpflichtung zu „allgemeiner und umfassender Abrüstung unter wirksamer internationaler Kontrolle", Ziel zahlreicher VN-Resolutionen, wurde ignoriert und die Bedeutung der UNO als effektive Weltfriedensorganisation in der Praxis immer stärker unterlaufen. Stattdessen avancierte Deutschland fast unbemerkt zum weltweit drittgrößten Rüstungsexporteur.

\section{Historischer Rückblick}

Den Weg zu einer friedlichen und gerechten, demokratischen Weltordnung hatten Politiker in Ost und West, die Schrecken des Zweiten Weltkriegs noch vor Augen, 1945 vorgezeichnet. Die VN-Charta sah in Artikel 106 vor, dass sich die Weltorganisation in einer Übergangsphase, in der die ständigen Mitglieder des Sicherheitsrats die Verantwortung für die Wahrung des Friedens übernehmen würden, zu einer kompetenten Sicherheitsbehörde und eventuell sogar zu einer föderalen Weltunion entwickeln würde. Damit ging die UNO in ihren Zielen weit über das hinaus, was bereits mit der Gründung des Völkerbunds erreicht worden war: Ein wirksames System kollektiver Sicherheit, an dem auch die Sowjetunion ein vitales Interesse hatte, sollte den Frieden weltweit sichern. Des Weiteren hatte sich der Sicherheitsrat, anders als es beim Völkerbund noch der Fall

5 Jost Delbrück, Deutschland und die Vereinten Nationen - Rückschau und Perspektiven, in: Ernst Koch (Hg.), Die Blauhelme. Im Einsatz für den Frieden. Frankfurt/M. und Bonn 1991, S. 215

6 Die Entscheidung wurde 1994 im „Out-of-Area“-Urteil des Bundesverfassungsgerichts bekräftigt. 
war, den Mitgliedern geöffnet, die ihm nun die Hauptverantwortung für die Wahrung des internationalen Friedens und der nationalen Sicherheit übertragen sollten ${ }^{7}$ Das moderne Friedensverfassungsrecht reflektierte und verstärkte diesen Trend und entsprach damit dem Erkenntnisstand und den Rechtsentwicklungen in vielen Teilen der Welt, vor allem aber in Europa, das diesbezüglich eine besondere historische Verantwortung wahrzunehmen hatte.

Es verwundert nicht, dass in Indien eine starke anti-imperialistische, gewaltfreie Bewegung für eine ,Welt ohne Waffen' entstanden war. Mahatma Gandhi und der indische Nationalkongress hatten bereits in der berühmten Quit India Resolution am 8. August 1942 gefordert: „Der zukünftige Friede, die Sicherheit und der geordnete Fortschritt der Welt erfordern eine Weltföderation der freien Nationen; auf keiner anderen Grundlage können die Probleme der modernen Welt gelöst werden. “Indien verfolgte nach Beendigung des Krieges so eine Außenpolitik, die sich ganz wesentlich an den Vereinten Nationen orientierte. In seiner Rede an das All India Congress Committee (AICC) unterstrich Gandhi: „Wir wollen nicht wie Frösche im Brunnen sein. Unser Ziel ist die Weltföderation. Sie kann nur durch [eine Politik der] Gewaltlosigkeit zustande kommen. "8 Die Quit India Resolution` wurde unter großem Beifall verabschiedet.

Nach dem Zweiten Weltkrieg setzte sich auch Großbritanniens Außenminister Ernest Bevin für ein Weltparlament ein, ${ }^{9}$ vielleicht auch in der Annahme, die Deutschen würden sich an ähnliche Vorschläge ihrer Delegation 1919 in Versailles erinnern. In Fernost hatte Japan in seiner Verfassung zukünftigen Kriegen und dem Krieg überhaupt eine Absage erteilt, in der Hoffnung, dass andere Staaten nachziehen und die Vereinten Nationen bald ihre friedenssichernden Aufgaben übernehmen könnten. ${ }^{10}$ In den USA waren 1949 in beiden Häusern des Kongresses Resolutionen verabschiedet worden - mit dem Ziel, die Entwicklung der Vereinten Nationen zu einer demokratischföderalen Weltunion mit beschränkten, aber definitiven gesetzgeberischen, rechtsprechenden und Exekutivvollmachten zu ermöglichen.

Warum hat es solche Äußerungen und Initiativen von offizieller Seite in Deutschland nicht gegeben? ${ }^{11}$ Hatten Realpolitiker,

7 Es verhält sich nicht etwa so, dass die VN-Mitgliedstaaten dem Sicherheitsrat bereits aufgrund ihrer Mitgliedschaft gemäß Artikel 24 der VN-Charta die Hauptverantwortung für die Wahrung des Weltfriedens und der internationalen Sicherheit übertragen haben. Wie auch der Völkerrechtler Knut Ipsen in einem Brief an den Verfasser bestätigt, würde „[E]twas derartiges [..] voraussetzen, daß die Staaten insoweit eine bis dato bestehende Kompetenz regelrecht aus ihrem eigenen Kompetenzbereich herausgelöst und der UNO zugeschoben haben [...]. Die Charta der Vereinten Nationen ist gewiß ein hochrangiger Vertrag [...], aber er ändert die Staatenkompetenzstruktur nicht mehr als jeder andere völkerrechtliche Vertrag" (Brief v. 19.4.1999). Eben das aber ist Sinn und Zweck des Artikels 24.

8 Speech at A.I.C.C. Meeting, Bombay, 7. August 1942, The Collected Works of Mahatma Gandhi (Electronic Book), New Delhi 1999, Bd. 83, S. 181-185. Online: http://www.gandhiserve.org/cwmg/VOL083.PDF. S. auch K. Schlichtmann, Mahatma Gandhi and the Quest for an Effective United Nations Organization, The Stakes, 1917-1947, Gandhi Marg, Vol. 26, No. 1 (April-Juni 2004), S. 55-81.

9 House of Commons Debate, in Foreign Affairs, Vol. 416 (23 November 1945), S. 785.

10 S. Klaus Schlichtmann, Die Abschaffung des Krieges. Artikel IX, Ursprung, Auslegung und Kontroverse, $S+F, 4 / 2002$, S. 223-229.

11 Ausnahmen gab es freilich, etwa Carlo Schmid. Er musste sich allerdings gegen den Vorwurf wehren, er vertrete undeutsche Interessen. Carlo Schmid, Legionäre fremder Interessen? Kollektive Sicherheit statt Remilitarisierung, Die Welt, 14.12.1948, und ders. Deutsche Soldaten in einer internationalen Wehrmacht - nicht als Legionäre fremder Interessen, Die Welt, 28.4.1949. wie der belgische Außenminister Paul-Henri Spaak, nicht vor einer „regionalen Blockpolitik, die im Gegensatz zur Politik der kollektiven Sicherheit stehen würde“12, gewarnt? Es mussten ja nicht gleich, wie in Indien, England, Japan oder den USA, die Regierungen bzw. Regierungsmitglieder sein, die sich für solche Ziele einsetzten.

Dabei hatte zwischen 1945 und 1955 die bundesdeutsche Friedensbewegung ähnliche, an die Weltbürgerbewegung anknüpfende Ziele verfolgt. ${ }^{13}$ Aber „die politische Wirklichkeit war [...] von der von Hegel beeinflussten machtstaatlichen ,Realpolitik' geprägt [...], die dem Gedanken einer ,internationalistischen “Friedensorganisation mit Skepsis begegnete." ${ }^{14}$ Und dass „auch nach dem Ende des 2. Weltkrieges die Aussichten für eine nachhaltige Einstellungsänderung [...] gegenüber einer friedenssichernden Weltorganisation nicht positiv [waren], “15 dafür sorgten die ,Seilschaften“ im Bonner Außenministerium. Dagegen sah das Grundgesetz eine Weltordnung vor, in der der Krieg geächtet und stattdessen ein System kollektiver Sicherheit unter der Ägide der Vereinten Nationen etabliert, dass Frieden ohne bzw. mit immer weniger Waffen garantieren würde. Doch die Diplomatie hierzulande stand offenbar in einer Tradition, die eine solche Ausrichtung ablehnte oder ignorierte. Dass auch in den folgenden 45 Jahren nichts geschah, die ursprünglichen Ziele der Vereinten Nationen zu realisieren, lag nicht nur am aggressiven, expansiven Weltkommunismus: Nachdem Deutschland zweimal einen totalen Krieg heraufbeschworen und die Teilung Europas selbst verursacht hatte, haben beide deutschen Staaten diesen Weg nach 1949 offensichtlich blockiert, indem sie, jeder auf seine Weise, beständig und kräftig das Feuer des Kalten Kriegs schürten.

\section{Föderale Weltunion als demokratische, globale Neuordnung}

Wie konnte die Bevölkerung dermaßen in die Irre geführt werden? Der Gesetzgeber ist verpflichtet, Schritte zu unternehmen, um das Verfassungsziel „kollektive Sicherheit“ auf den Weg zu bringen. Die Unfähigkeit bzw. Unterlassung aller bisherigen Bundesregierungen, das grundgesetzliche Friedensgebot im Hinblick auf die Staatszielbestimmung kollektive Sicherheit in praktische Politik umzusetzen, ist beklagenswert. ${ }^{16}$

Dabei haben sich zahlreiche europäische Staaten der friedenssichernden Weltorganisation verpflichtet, indem sie in ihren Verfassungen eine entsprechende Souveränitätseinschränkung oder -übertragung vorsehen. Sie haben erkannt, dass ein relativer Verlust an Souveränität einen Gewinn an Sicherheit bringen und zur Lösung der nur gemeinsam zu bewältigenden Probleme notwendig beitragen muss. ${ }^{17}$ Mit anderen Worten:

12 N.Y. Times, 19.11 .44 (eigene Übersetzung).

13 Klaus Schlichtmann, Helmut Hertling und die Weltbürgerbewegung, in Detlev Bald und Wolfram Wette (Hg.), Friedensinitiativen in der Frühzeit des Kalten Krieges 1945-1955, Essen 2010, S. 155-174.

14 Delbrück, Deutschland und die Vereinten Nationen, a.a.O., S. 212.

15 Ebd., in Ernst Koch, S. 13.

16 Artikel 24 Absatz 1 ist zwar eine Kann-Bestimmung. In einer Situation, in der der Weltfriede und die internationale Sicherheit akut gefährdet sind, kann der Bundestag sich jedoch seiner verfassungsmäßigen Bestimmung, dem Frieden zu dienen, nicht entziehen.

17 Vgl. auch die bereits vor 100 Jahren angestellten Überlegungen von Walther Schücking, Die Organisation der Welt, Berlin 1908. 
Die Europäer können den VN genau die Kompetenzen „durch Gesetz“ übertragen, die die Weltorganisation braucht, um umfassende Abrüstung zu organisieren und in Krisensituationen wirksam und schnell handeln zu können. Das geht jedoch offenbar nur, wenn die ,Nummer eins‘ in Europa, die Bundesrepublik Deutschland, den Anstoß gibt. Nach dem Ius-cogens-Gesetz der obligatio erga omnes ${ }^{18}$ und dem Reziprozitätsgrundsatz müssten dann alle Staaten in der Folge ebenfalls in entsprechende Hoheitsbeschränkungen einwilligen und sich bedingungslos der Rechtsprechung des Internationalen Gerichtshofs (IGH) unterwerfen.

Vielleicht im Hinblick und in der Hoffnung darauf, dass Deutschland seiner friedenspolitischen Bestimmung nach der Wiedervereinigung nachkommen würde, hatte der damalige französische Staatspräsident François Mitterrand im Januar 1992 vorgeschlagen, die französische Nuklearstreitmacht den gemeinsamen Interessen der Europäischen Gemeinschaft unterzuordnen. Damit eröffnete die französische Initiative die Möglichkeit einer Zusammenlegung der beiden europäischen Sicherheitsratssitze zu einem gemeinsamen Sitz. Das Angebot war gekoppelt mit dem Vorschlag, den VN Truppen zur Verfügung zu stellen, die dem Generalstabsausschuss der Vereinten Nationen unterstellt sein sollten. ${ }^{19}$ Auch die Sowjetunion hatte anlässlich der Golfkrise 1990 den Einsatz einer gemeinsamen Flotte unter der Flagge der Vereinten Nationen und die Aktivierung des VNGeneralstabs vorgeschlagen. ${ }^{20}$ In Deutschland ging der Trend in eine andere Richtung.

Die Bundesrepublik hätte nach der Wiedervereinigung „ein Zeichen [...] setzen und die Anerkennung Sloweniens und Kroatiens, aber auch der Ukraine und anderer neuer Staaten, an die Bedingung knüpfen [müssen], dass diese Staaten sich der Rechtsprechung des Internationalen Gerichtshofs (IGH) in Den Haag unterwerfen" . ${ }^{21}$ In der Regel sollte, wie bereits auf den Haager Friedenskonferenzen gefordert, jeder zivilisierte Staat, der die volle Souveränität erlangt hat oder im Begriff steht, diese zu erlangen, sich ohne Weiteres der Rechtsprechung des IGH unterwerfen, um damit seinen Willen zur friedlichen Streitbeilegung, und endlich: Abschaffung der Institution des Krieges, zu bekunden. Dass dieser Mechanismus nach dem Zweiten Weltkrieg nicht in dem Maße in Anspruch genommen wurde, wie ursprünglich von den Vereinten Nationen vorgesehen, hat verschiedene Gründe. Nach 1945 hatte nicht nur Westdeutschland, sondern auch der gesamte Ostblock sich geweigert, eine Unterwerfungserklärung gemäß Art. 36 Abs. 2 des Statuts des IGH abzugeben und die Rechtsprechung des IGH als obligatorisch anzuerkennen. Eine solche Erklärung beinhaltet einen unangenehmen Souveränitätsverzicht, der zusätzliche Schritte

$18 \mathrm{Zu}$ den Grundsätzen der obligatio erga omnes und actio popularis siehe Yasuhiko Saito, International Law as a Law of the World Community - World Law as Reality and Methodology, in: Atle Grahl-Madsen and Jiri Toman (Hg.), The Spirit of Uppsala, Proceedings of the Joint UNITAR-Uppsala University Seminar on International Law and Organization for a New World Order (JUS 81), Uppsala 9-18 June 1981, Berlin and New York 1984, S. 233-49.

19 Paul Zaylor, France might share its bomb, Japan Times, 15 January 1992; H. Peter Langille, Conflict Prevention: Options for Rapid Deployment and UN Standing Forces, in: Oliver Ramsbotham and Tom Woodhouse, (Hg.), Warlords, Hawks and Doves: Peacekeeping as Conflict Resolution, London 2000.

20 Roland Dore, Japan, Internationalism and the UN, London und New York 1997, S. 90.

21 So eine Forderung des Verfassers Ende 1991 in einem Brief an den damaligen deutschen Außenminister Hans-Dietrich Genscher (Schreiben vom 9.12.1991) erfordert, wenn es darum geht, ein funktionierendes System kollektiver Sicherheit und eine gewisse supranationale Exekutivgewalt der Vereinten Nationen zu begründen. Stattdessen trat Deutschland 1955 dem Nordatlantischen Verteidigungsund Militärbündnis (NATO) bei.

Die Bundesrepublik war schlecht beraten, sich an die Spitze derer zu stellen, die ein schnelles und wirksames Handeln der Vereinten Nationen einseitig auf Macht und militärische Gewalt gründen wollten. Selbst wenn die VN-Charta regionale Bündnisse, auch Verteidigungsbündnisse, zulässt, so ist die Zeit, da diese Bündnisse die regionale Sicherheit garantieren und auf eine wirksame, mit ausreichenden Vollmachten ausgestattete Weltorganisation verzichten können, in der Ära atomarer Bedrohung endgültig vorbei. Die Erfolglosigkeit der Bemühungen in den vergangenen zwei Jahrzehnten, den Frieden militärisch zu sichern, sollte zu denken geben.

Allerdings hat die deutsche Ablehnung eines auf Rechtsprinzipien gegründeten Friedens Geschichte. Schon die Haager Friedenskonferenzen 1899 und 1907 waren gescheitert, weil Deutschland die Schaffung eines Internationalen Gerichtshofs mit verbindlicher Rechtsprechung und Abrüstungsverpflichtungen mit einem Veto belegte. Die große Mehrheit der teilnehmenden Staaten hatte mit den damaligen Mächten England, Frankreich, den USA, Russland und China für die „obligatorische Schiedsgerichtsbarkeit “ gestimmt. Es überrascht nicht, wenn eben diese Staaten, die im Ersten Weltkrieg auf Seiten der Entente gekämpft und den Völkerbund vorbereitet hatten, heute einen Ständigen Sitz im Sicherheitsrat einnehmen, um für die für einen dauerhaften, gesicherten Frieden unerlässliche Übergangszeit Verantwortung zu übernehmen.

Für die deutschen Versäumnisse und Verfehlungen gibt es nur eine schlüssige Erklärung: Das Auswärtige Amt, unterstützt von einer akademischen Elite, die einer Tradition huldigt, die dem Pazifismus kritisch bis ablehnend gegenübersteht, hat bislang die Umsetzung des Friedensgebots verhindert. ${ }^{22}$ So war auch nach dem Zweiten Weltkrieg die Bundesrepublik über 60 Jahre lang nicht bereit, sich zur friedlichen Beilegung zwischenstaatlicher Streitigkeiten der Rechtsprechung des Internationalen Gerichtshofs zu unterwerfen, obwohl das Grundgesetz dies im Art. 24 Abs. 3 verbindlich vorschreibt. Erst 2008 hat sie eine entsprechende Erklärung abgegeben, die jedoch durch den doppelten „Streitkräfte-Vorbehalt“ letztendlich wertlos und wirkungslos geblieben ist. $^{23}$

\section{Ist ein Durchbruch in Sicht?}

Die ständigen Sicherheitsratsmitglieder (P5) können nur wenig dazu beitragen, die VN mit hoheitlichen Kompetenzen auszustatten, da sie bereits selbst Kern und Garant der zukünftigen

22 Eckart Conze, Norbert Frei, Peter Hayes und Moshe Zimmermann, Das Amt und die Vergangenheit. Deutsche Diplomaten im Dritten Reich und in der Bundesrepublik, München 2010. Kritische Zusammenfassung bei Richard J. Evans, The German Foreign Office and the Nazi Past, Neue Politische Literatur, Jg. 56 (2011), S. 165-183.

23 Siehe dazu u.a. die Stellungnahmen der deutschen Sektion der International Association of Lawyers Against Nuclear Arms (IALANA), http://ialana.de/ agenda/voelkerrecht/anerkennung-gerichtsbarkeit-igh. Vgl. Dieter Deiseroth, Internationale Gerichtsbarkeit und friedliche Streitbeilegung, Wissenschaft \& Frieden, 1/2000. 
Weltordnung sind. Sie entscheiden jedoch über Einsatz und Zusammensetzung der friedenssichernden und friedenserhaltenden Truppen. Gemeinsam könnten die „P5“ auch ohne das Legitimationsmäntelchen der UNO-Charta oder in einer Koalition der Willigen tätig werden.

Allerdings sind die VN-Friedenstruppen bekanntlich nur temporäre Einrichtungen. Sie legitimieren aber indirekt - und ohne dass dies beabsichtigt ist - das Fortbestehen des militärisch-industriellen Komplexes. Kollektive Sicherheit dagegen impliziert, dass die Staaten Streitigkeiten ausschließlich auf dem Rechtswege klären und eine rechtsverbindliche internationale Ordnung Frieden und Sicherheit gewährt. Die VN-Charta schließlich verbietet Krieg in unmissverständlicher Weise.

Funktionieren und Einsatz der in der Charta vorgesehenen VNPolizeitruppen ${ }^{24}$ setzen voraus, dass der in Kapitel VII vorgesehene Generalstabsausschuss des Weltsicherheitsrats ermächtigt sowie nach Art. 26 der Charta ein funktionierendes System der Rüstungsregelung in Kraft ist, und das hieße konkret: wirksame Rüstungskontrolle und Abrüstung auf ein für den Grenzschutz erforderliches Minimum. Beides ist auch 20 Jahre nach dem Ende des Kalten Kriegs noch immer nicht gegeben.

Die US-amerikanische Politik für das Dilemma verantwortlich zu machen, ist wenig hilfreich und enthebt uns nicht unserer eigenen Verantwortung.

Die notwendige, längst überfällige Trendwende in der deutschen Außenpolitik fordert einen grundlegenden Gesinnungswandel. Die vorgeschlagenen, im Friedensvölkerrecht und in den Verfassungen verankerten Bestimmungen sind Ergebnis irreversibler historischer Entwicklungen. Um eine neue, an friedenspolitischen Maximen orientierte Außenpolitik zu begründen, wird man zudem nicht umhin kommen, über die in unseren Schul- und Lehrbüchern nicht hinreichend erklärten Ziele und Inhalte der Haager Friedenskonferenzen aufzuklären.

\section{Aktive Gewaltfreiheit}

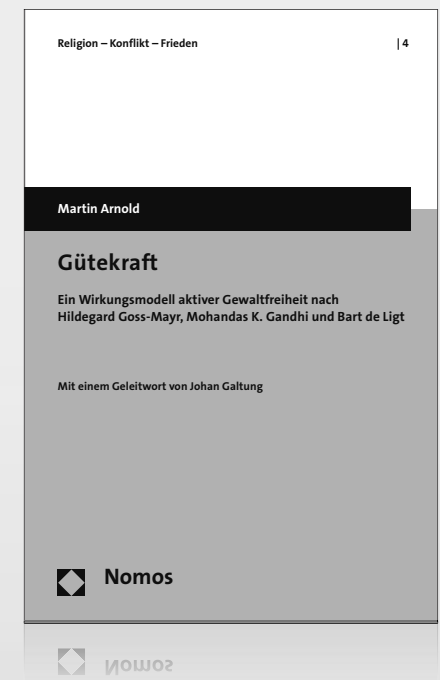

Gütekraft

Ein Wirkungsmodell aktiver Gewaltfreiheit nach Hildegard Goss-Mayr, Mohandas K. Gandhi und Bart de Ligt

Mit einem Geleitwort von Johan Galtung

Von Martin Arnold

2011, 283 S., brosch., 19,- €, ISBN 978-3-8329-6975-2

(Religion - Konflikt - Frieden, Bd. 4)

Das Gütekraft-Modell beschreibt den allgemeinmenschlichen Kern erfolgreicher Ansätze zur gewaltfreien Lösung von Unrechts- und Gewaltsituationen, von der persönlichen Ebene bis zu globalen Konflikten.

"Arnold hat jene wirksame Kraft von einem allgemeinmenschlichen Hintergrund aus erforscht, und es ist ihm gelungen, sie ohne Rückgriff auf eine bestimmte Religion oder Philosophie darzustellen."

Fromm Forum 16/12

Weitere Informationen: www.nomos-shop.de/14083

Nomos

24 Karlheinz Koppe, Argumente für Aufbau und Einsatz einer internationalen Polizei, Friedensforum 4 (2005). 